\title{
Size dependence investigations of hot electron cooling dynamics in metal/adsorbates nanoparticles
}

\author{
Christophe Bauer *, Jean-Pierre Abid, Hubert H. Girault \\ Laboratoire dElectrochimie Physique et Analytique, Faculté des Sciences de Base, Ecole Polytechnique Fédérale de Lausanne, \\ CH-1015, Lausanne, Switzerland \\ Received 13 December 2004; accepted 15 June 2005 \\ Available online 28 July 2005
}

\begin{abstract}
The size dependence of electron-phonon coupling rate has been investigated by femtosecond transient absorption spectroscopy for gold nanoparticles (NPs) wrapped in a shell of sulfate with diameter varying from 1.7 to $9.2 \mathrm{~nm}$. Broad-band spectroscopy gives an overview of the complex dynamics of nonequilibrium electrons and permits the choice of an appropriate probe wavelength for studying the electron-phonon coupling dynamics. Ultrafast experiments were performed in the weak perturbation regime (less than one photon in average per nanoparticle), which allows the direct extraction of the hot electron cooling rates in order to compare different NPs sizes under the same conditions. Spectroscopic data reveals a decrease of hot electron energy loss rates with metal/ adsorbates nanosystem sizes. Electron-phonon coupling time constants obtained for $9.2 \mathrm{~nm}$ NPs are similar to gold bulk materials ( $\sim 1 \mathrm{ps})$ whereas an increase of hot electron cooling time up to $1.9 \mathrm{ps}$ is observed for sizes of $1.7 \mathrm{~nm}$. This is rationalized by the domination of surface effects over size (bulk) effects. The slow hot electron cooling is attributed to the adsorbates-induced long-lived nonthermal regime, which significantly reduces the electron-phonon coupling strength (average rate of phonon emission).
\end{abstract}

(C) 2005 Elsevier B.V. All rights reserved.

Keywords: Electron-phonon coupling; Metal; Adsorbates; Surface femtochemistry; Size behavior; Nanoparticles; Ultrafast spectroscopy; Nonthermal regime

\section{Introduction}

Electron transfer (ET) reactions are the most basic processes in chemistry and are ubiquitous in physics and biology [1]. ET processes can be divided in two classes: (i) homogeneous ET and (ii) heterogeneous ET. Homogeneous ET mainly deals with ET in solution and is very well understood with the Marcus theory. On the opposite, the understanding of heterogeneous ET lies far behind the analogous homogeneous problem despite the observation that most of efficient natural and technological chemical transformation occurs at interfaces. ET processes underlying redox reactions between

\footnotetext{
* Corresponding author.

E-mail address: christophe.bauer@epfl.ch (C. Bauer).
}

a metal electrode and a molecule in solution represents the heart of electrochemistry [2]. The lack of a clear picture for heterogeneous ET comes from the technical difficulties to investigate interfacial or surface phenomena [3]. Furthermore, the interfacial dynamical phenomena may be more complex than in homogeneous media due to the appearance of novel energy dissipation channels.

The purpose of the present study is to obtain information on the dynamical behavior of nonequilibrium electrons at the metal/molecule interface with a particular interest here for the electron-phonon coupling. In other words, we would like to know how a bidirectional ET process between adsorbates and metal can affect the global dynamics of the nanosystem. Indeed, metal/ adsorbates NPs under investigations here exhibit 
ultrafast back and forth charge resonance tunneling between metal and empty electronic states of adsorbates. Dynamical events occurring at the metal/adsorbates junction play a crucial role for a wide range of applications [4], like for example, surface femtochemistry [5], catalysis [6], manipulations with scanning tunneling microscope tips [7], organic light emitting devices [8,9], surface enhanced Raman scattering [10], and molecular electronic devices [11,12].

\subsection{Nonequilibrium electron relaxation processes in metal}

Let us first describe the behavior of nonequilibrium electrons in bulk metal. The classical picture of the different events of nonequilibrium electron relaxation occurring in metal involves three main processes which are illustrated in Fig. 1A: (i) electron-electron scattering $\left(\mathrm{P}_{\mathrm{I}}\right)$, (ii) electron-phonon interaction $\left(\mathrm{P}_{\mathrm{II}}\right)$ and (iii) phonon-medium interaction $\left(\mathrm{P}_{\mathrm{III}}\right)$. The perturbation of the electron distribution upon absorption of a femtosecond laser pulse by a metal leads to the creation of a population of nascent nonthermal electrons (NNEs). In bulk metal, NNEs redistribute the photonic energy by e-e scattering with free "cold" conduction electrons giving rise to a well-defined temperature of the hot electron gas (internal thermalization process $\mathrm{P}_{\mathrm{I}}$ ). The electronic temperature rise varies from hundreds to thousands of Kelvin depending on the laser pump fluence. The hot electron gas transfers the energy toward the lattice by electron-phonon interaction leading to a thermal equilibrium between the hot electron gas and the lattice. This process is referred to the external thermalization process $\mathrm{P}_{\mathrm{II}}$, which is also called hot electron cooling and is the main topic of this work. In the last step, the phonons transfer their energy to the surrounding medium (heat dissipation process $\mathrm{P}_{\mathrm{III}}$ ) within hundreds of picoseconds. All these processes of electron and energy redistribution are not necessary sequential but may overlap in time. Due to the diversity of the terms to describe these phenomena, a note is given to present the equivalences [13].

\subsection{The two-temperature model}

The two-temperature model (TTM), which is the most commonly employed model to describe hot electron cooling process divides the problem into two subsystems [14,15]. The first subsystem is represented by the population of hot electrons defined by an electronic temperature $T_{\mathrm{e}}$ whereas the second one is linked the population of phonons defined by the lattice temperature $T_{1}$. Within the TTM, the rate of energy exchange between the hot electron gas and the lattice is given by two dependent differential equations (Eqs. (1a) and (1b)) with $g$, the electron-phonon coupling constant, $C_{\mathrm{e}}$ and $C_{\mathrm{l}}$, the electronic and the lattice heat capacity, respectively

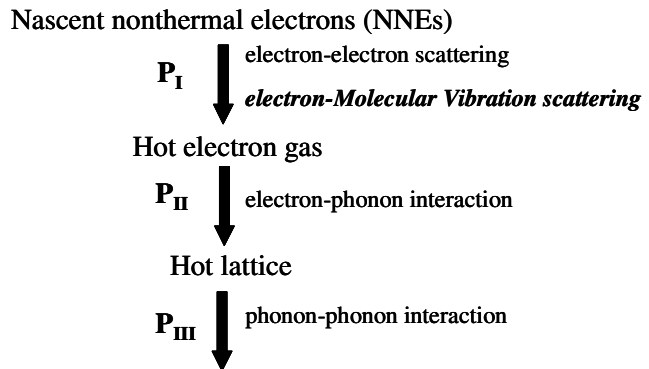

A Surrounding medium

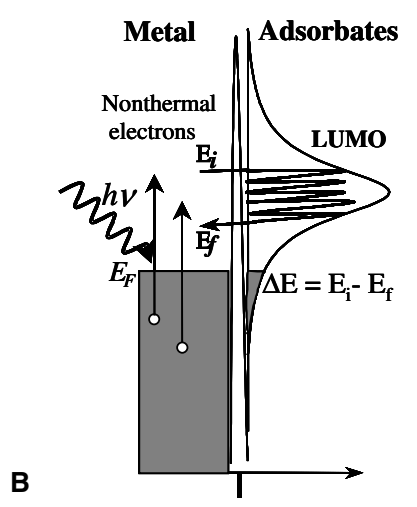

Fig. 1. (A) Schematic diagram of the different steps of photonic energy redistribution in metal NPs. Nascent nonthermal electrons created upon the absorption of a femtosecond laser pulse dissipate the excess energy among free electrons via e-e scattering and leads the formation of a hot electron gas with a well defined electronic temperature, the process $P_{I}$ is called internal thermalization and corresponds to the build-up of a Fermi-Dirac distribution. The electron-molecular vibration scattering is a process specific to the adsorbates/metal systems and occurs via a bidirectional inelastic electron tunneling into adsorbates (see B). During the external thermalization $\left(\mathrm{P}_{\mathrm{II}}\right)$, the hot electron gas transfers the energy to the lattice through electronphonon coupling. Finally, the last process corresponds to the heat dissipation toward the surrounding medium via phonon-medium coupling $\left(\mathrm{P}_{\mathrm{III}}\right)$. (B) Schematic picture showing the inelastic resonant scattering of nonequilibrium electrons into empty electronic states of adsorbates. Immediately after the absorption of the femtosecond pulse, a nonequilibrium distribution of nascent nonthermal electrons (as photoexcited) is created with energies $E_{\mathrm{i}}$ in the range $E_{\mathrm{F}} \leqslant E_{\mathrm{i}}<$ $E_{\mathrm{F}}+h v$ for intraband transitions. The nonequilibrium electron is temporary transferred into an empty electronic level with an energy $E_{1}$ with the subsequent creation of an intermediate negative ion state, the lifetime of this state is very short on the order of tens of femtoseconds. The electron then scatters back with an energy $E_{\mathrm{F}}$ into the gold metal conduction band. The nascent nonthermal electrons have deposited energy $\Delta E=E_{\mathrm{i}}-E_{\mathrm{F}}$ in the affinity electronic levels of adsorbates and is converted in molecular vibrational energy. The amount of energy loss into adsorbates is directly related to the hot electron resident lifetime.

$C_{\mathrm{e}}\left(T_{\mathrm{e}}\right) \frac{\partial T_{\mathrm{e}}}{\partial t}=-g\left(T_{\mathrm{e}}-T_{1}\right)$,

$C_{1} \frac{\partial T_{1}}{\partial t}=g\left(T_{\mathrm{e}}-T_{1}\right)$.

The TTM implies that the metal nanoparticle system with the two subsystems $T_{\mathrm{e}}$ and $T_{1}$ can be seen as a closed system. In other words, energy can only be exchanged 
between two subsystems represented by the populations of hot electron and phonons since the energy transfer toward the surrounding medium is not taken into account within these assumptions. On the other hand, from a temporal point of view, the TTM assumes that the electronic temperature can be defined at any time of the process of nonequilibrium electrons relaxation. This assumption implies that the internal thermalization time (e-e scattering time) can be neglected with respect to the cooling time of hot electrons $\left(\tau_{\mathrm{e}-\mathrm{e}} \ll \tau_{\mathrm{e}-\mathrm{ph}}\right)$. However, recent experiments [16,17] and theoretical models [18] have highlighted the fact that under certain conditions, the dynamics of nonequilibrium electrons can quit the frame of the TTM [19]. Indeed, it appears that e-e scattering time cannot be neglected with respect to e-ph coupling time when adsorbates affect the nonequilibrium electron dynamics.

\subsection{Surface femtochemistry}

When molecules are chemisorbed on metal surfaces, additional energy loss channels for nonequilibrium electrons may appear. A part of the photonic energy can be transferred to the adsorbates instead of heating up the bulk lattice by electron-phonon coupling. The so-called field of surface femtochemistry deals with chemical reactions induced on metal surfaces by photoexcited electrons created in the substrate $[5,20,21]$. Fig. 1B illustrates the primary steps of energy transfer from the metal toward the adsorbates. The photoexcited nonequilibrium electrons experience an inelastic resonant scattering process into empty electronic states of adsorbate to form a transient negative ion, which accumulates kinetic energy while the evolution of the wave packet on potential energy surface. After a certain resident time, nonequilibrium electrons scatter back into metal leaving behind "hot" adsorbates (highly vibrationally excited adsorbates). If the kinetic energy deposited into adsorbates by nonequilibrium electrons is high enough, chemisorbed species can either desorb or dissociate through the MGR [22,23] or Antonivietz model [24]. Usually, the hot electron gas is thought to be the only nature of photoexcited electrons, which induce desorption or dissociation via inelastic resonant scattering. Indeed, the description of the interaction of nonequilibrium electrons with LUMO of adsorbates with the TTM draws outside the possible implication of nonthermal electrons in the process of inelastic electron tunnelling since the population of nonthermal electrons has disappeared when an electronic temperature is established. However, nonthermal electrons may also drive surface chemistry as proposed from the desorption yield dependence on excitation wavelength $[25,26]$, from long-lived nonthermal electrons [17] or from the direct observation by transient absorption spectroscopy [19].
1.4. Technical approaches to investigate interfacial charge transfer processes

In the past, studies on interfacial ET between metal and molecules were mainly performed by electrochemical methods. This steady-state approach only allows the determination of the rate-limiting step and the fastest processes remain elusive. Time-resolved optical spectroscopic techniques permit obtaining real-time information on heterogeneous ET reactions. While most of the ultrafast investigations on adsorbates/metal systems (surface femtochemistry) have been performed with time-resolved two-photon photoemission (a nonoptical technique) studies with optical spectroscopic methods are poorly known. Indeed, ultrafast optical spectroscopy is difficult to perform on metal/molecule interfaces due to the very weak signal arising from a monolayer of adsorbates. However, the use of nanoparticles covered by a monolayer of adsorbates allows the study of electronic surface phenomena by overcoming this technical problem since the huge surface-to-volume ratio of NPs permits the enhancement of the signal hundreds of times compared to a metal film covered by a monolayer of adsorbates.

\subsection{Size dependence investigations on electron-phonon coupling rate in metal NPS}

Although the electron-phonon interaction process has been extensively studied in metal nanoparticles [2731], there are still controversies for size dependence behavior on hot electron cooling dynamics in metal NPs. These discrepancies likely come from the difficulties to extract electron-phonon coupling constants and/or from different experimental conditions. Size independent e-ph coupling constants have been reported for Au NPs sizes from 2 to $8 \mathrm{~nm}$ [32]. These results are understood by the domination of bulk electron-phonon coupling constant due to weak interaction between hot electron gas and surface phonon modes [32]. Ahmadi et al. [33,34] also observed independent hot electron cooling times for 1.9-50 nm gold NPs within the high perturbation regime. As it will be discussed below, the dependence of hot electron cooling rate on pump fluence makes difficult a direct comparison between NPs sizes. Very recently, Arbouet et al. [35] reported size dependent electronphonon scattering times in Ag and Au NPs for sizes below $10 \mathrm{~nm}$, which has been rationalized by a reduction of the screening electron-ion interaction due to a spill-out effect. The acceleration of the hot electron cooling process as the NPs size decrease is caused by a confinement (size) effect linked to metal bulk properties. These reports dealt with the so-called "naked metal NPs", that means either metal NPs without adsorbates or a situation where the surrounding medium (matrix) does not affect nonequilibrium electron dynamics. Several studies have 
shown that the surrounding medium strongly influences the dynamics of hot electron cooling within the strong perturbation regime [36-38]. Furthermore, the chemical nature of adsorbates can also affect the electron-phonon coupling rates in gold core-shell NPs as reported by Westcott et al. [39]. Up to now, investigations on size dependence of nonequilibrium electron dynamics in adsorbates/metal NPs cannot be found in the literature.

\subsection{Nonequilibrium electron dynamics in metall adsorbates nanosystems}

For the present size dependence investigation, we have prepared metal/adsorbates systems from 1.7 to $9.2 \mathrm{~nm}$ where the ratios of surface metal atoms to total metal atoms vary from $73 \%$ to $13 \%$, respectively. The study is focused on electron-phonon coupling dynamics, the energy loss relaxation processes of hot electron gas. However since the different dynamical processes are interdependent and may overlap in time, let us present the knowledge available at the moment for these systems Previous ultrafast transient absorption measurements have shown that nonthermal electrons (NNEs) experience an inelastic resonant scattering process into empty electronic states of adsorbates in small metal NPs wrapped in a shell of adsorbates [19]. More strictly speaking, when chemical interface damping provides the main mechanism for the surface plasmon damping, ultrafast chemical interface scattering (UCIS) has been recognized as an important channel of energy and momentum loss for NNEs [19]. As a result of UCIS process, internal thermalization (build-up of a FermiDirac, i.e., establishment of electronic temperature) is slowed down and this effect is even more pronounced as NPs sizes decrease below $5 \mathrm{~nm}$ [40]. The mechanism behind the retardation of internal thermalization is beyond the scope of this paper. However, it could be useful to state here that this mechanism is directly related to surface effects, i.e., properties of adsorbates. In this paper, results obtained by ultrafast transient absorption spectroscopy on size behavior of hot electron cooling dynamics are presented for metal/adsorbates nanosystems in the critical size range below $10 \mathrm{~nm}$. Furthermore, these systems provide information as to how internal thermalization influences hot electron cooling process (electron-phonon interaction).

\section{Experimental}

\subsection{Samples preparation}

The three dimensional $\mathrm{Au} / \mathrm{TiO}_{2}$ nanoarchitectures were prepared by wet-grafting of initial Au NPs of $1.7 \pm 0.4 \mathrm{~nm}$ in diameter derivatized with mercapto succinic acid (MSA) to a $\mathrm{TiO}_{2}$ mesoporous thin film. The elementary $\mathrm{TiO}_{2}$ particle size is $15 \mathrm{~nm}$ and the thickness of the mesopourous films measured by profilometry is $5 \mu \mathrm{m}$. Before the insertion of gold NPs, the $\mathrm{TiO}_{2}$ nanocrystalline films exhibit a porosity of $60 \%$ with an average pore size of $20 \mathrm{~nm}$. Aqueous solution of primary $1.7 \mathrm{~nm}$ gold NPs were synthesized by $\mathrm{NaBH}_{4}$ reduction of $\mathrm{AuCl}_{4}^{-}$in a mixture of water and methanol. The synthesis includes five consecutive washing and centrifugation steps with pure methanol. This sequence ensures the removal of inorganic ions. The solvent excess was evaporated at temperatures below $40^{\circ} \mathrm{C}$ and a pressure lower than $5 \times 10^{-3}$ Torr for $12 \mathrm{~h}$. The core size of the resulting particles exhibited an average diameter of $(1.7 \pm 0.4) \mathrm{nm}$. The final gold NPs used to perform laser spectroscopic measurements was obtained by heat treatment at $723 \mathrm{~K}$ generating gold NPs wrapped in a shell of sulfate with sizes of $2.5-9.2 \mathrm{~nm}$ in diameter depending on wet-grafting time. The shell of sulfate results from the complete oxidation of the molecular bridge (mercapto succinic acid) as revealed by FT-IR spectroscopy and X-ray photoemission spectroscopy. The sizes of the gold NPs have been revealed by transmission electronic microscopy using a Philips CM20. For each NPs system, hundreds of NPs are counted resulting in a size distribution of 15-20\%. Full details concerning the preparation and the characterization will be published elsewhere. Finally, adsorbates/metal nanosystems under investigation consist of gold NPs wrapped in a shell of sulfate and embedded in a matrix of $\mathrm{TiO}_{2}$.

\subsection{Steady-state spectroscopy}

UV/Visible spectra were recorded on an HP-8353 (Hewlett-Packard) spectrophotometer.

\subsection{Femtosecond laser set-up}

The femtosecond transient absorption set-up features a compact CPA-2001 Ti:Sa-amplified femtosecond laser (Clark-MXR) with a repetition rate of $1 \mathrm{kHz}$, a fundamental wavelength at $775 \mathrm{~nm}$, a pulse width of $120 \mathrm{fs}$ and an energy of $1 \mathrm{~mJ}$ per pulse [41]. The output beam was split into three parts for pumping two double-stage noncollinear optical parametric amplifiers (NOPA) and to produce a white light continuum in a $2 \mathrm{~mm}$ thick sapphire plate. The output pulses of the NOPAs were compressed in SF10-glass prism pair compressors to obtain pulses of $50 \mathrm{fs}$. Pump and probe beams were directed parallel to each other toward a $60^{\circ}$ off-axis parabolic mirror that focused them into the sample. The resulting spot area of the pump pulse is $1.25 \times 10^{-4} \mathrm{~cm}^{2}$. The pump-probe cross-correlation measured by Kerr gating in a $0.3 \mathrm{~mm}$ thick PBH21 glass window (Ohara, Japan) at the sample position was typically $80 \mathrm{fs}$. 
For the monochromatic probing, a DET-110 silicon diode (Thorlabs) was placed directly after the samples to measure the transmitted light intensity. The signal from the detector was connected to a SR-830 lock-in amplifier (Stanford Research Instruments) tuned at $220 \mathrm{~Hz}$ by a chopper modulating the pump beam. Time delays between pump and probe pulses were controlled with a linear translation stage (Physic Instrument M511).

Transient absorption spectra were obtained using a white light continuum produced in a $2 \mathrm{~mm}$ thick sapphire plate. The white beam was collimated using a $90^{\circ}$ off-axis parabolic mirror and steered to the sample using only reflective optics. A smooth monotonic spectral distribution between 470 and $680 \mathrm{~nm}$ was obtained by controlling the pump energy with an iris and a variable density filter. A broad-band membrane beam splitter was placed before the sample to split the white light beam into signal and reference arms. Both were collected by fiber bundles and sent into a Triax 320 spectrograph (Jobin-Yvon). Reference and signal beams were separated vertically during their travel in the spectrograph and detected individually by diode array detector (A ST116 double Princeton Instrument). Both 1024 elements detector arrays allowed for recording more than $200 \mathrm{~nm}$ wide spectra averaging at a time. Chirped pulse duration was 210 fs as measured by Kerr gating in $\mathrm{PBH} 21$ glass between $490(2.53 \mathrm{eV})$ and $700 \mathrm{~nm}(1.77 \mathrm{eV})$. The group velocity dispersion of the white light continuum was compensated in the transient absorption spectra. The absorbance change was calculated using the ratio between data obtained with and without the pump pulses reaching the samples and corrected for fluctuations in the white light continuum intensity using the reference beam spectra. The low repetition rate of $1 \mathrm{kHz}$ of the present femtosecond set-up ensures that there is no continuous heating up during measurements due to a possible incomplete heat dissipation before the arrival of the second pump laser pulse.

\section{Results and discussion}

\subsection{Steady-state UVIVis spectra}

Fig. 2 shows the UV/Vis spectra of gold NPs wrapped in a shell of adsorbates with core metal sizes varying from 1.7 to $9.2 \mathrm{~nm}$. The increase of absorption below $450 \mathrm{~nm}$ is due to interband transitions from the $d$-band to $s p$-band of gold as well as band gap transition from $\mathrm{TiO}_{2}$ matrix. The manifestation of confinement in metal nanoparticles is characterized by the presence of surface plasmon, which corresponds to the collective oscillations of conduction electrons $[42,43]$. The surface plasmon bands are observed between 525 and $545 \mathrm{~nm}$ depending on metal/adsorbates nanosystem sizes. As

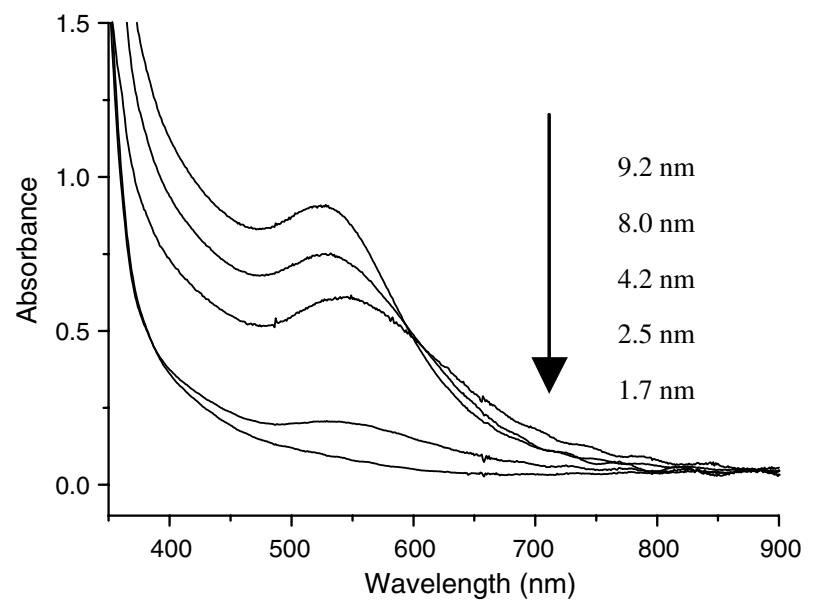

Fig. 2. Steady-state UV-Vis spectra of metal/adsorbates nanosystem with core diameter of $1.7,2.5,4.2,8$ and $9.2 \mathrm{~nm}$. Interference patterns arise from the homogeneous thickness of the $\mathrm{TiO}_{2}$ nanocrystalline film.

the NPs sizes decrease, the surface plasmon band becomes broader due to the scattering of collective electrons with NPs surface [44]. Indeed when the NP size is below the mean free path of bulk gold, surface scattering acts as an efficient damping mechanism for the surface plasmon. The electron scattering frequency $\Gamma$ (surface plasmon lifetime) is defined with Eq. (2) with A the damping constant, $\Gamma_{0}$, the bulk damping constant, $v_{\mathrm{F}}$ is the Fermi velocity of electrons and $R$ is the nanoparticles radius $[42,44]$

$\Gamma=\Gamma_{0}+\frac{A v_{\mathrm{F}}}{R}$.

\subsection{Chemical interface damping}

However with the presence of adsorbates on the NPs surfaces, an additional process for the damping of the surface plasmon, which is called the chemical interface damping may arise [42,45-47]. When adsorbates interact strongly with metal nanoparticles surface, electrons from metal NPs may tunnel back and forth into empty electronic states of the adsorbates [45]. The resident time of electrons in the adsorbates is long enough to break the plasmon phase coherence, leading therefore to a decrease of surface plasmon lifetime [45]. Thus, the term $A$ should be divided into two terms ( $A=$ $\left.A_{\text {size }}+A_{\text {adsorbates }}\right)$ when the chemical interface damping strongly contributes to the surface plasmon damping. The parameter $A$ contains contribution from both pure size (bulk) effects $A_{\text {size }}$ as well as the chemical interface damping $A_{\text {adsorbates }}$. The term $A_{\text {size }}$ depends on metal bulk properties whereas $A_{\text {adsorbates }}$ is related to the chemical environment directly in contact with the metal nanoparticles surface, i.e., the adsorbates. Strictly speaking, $A_{\text {adsorbates }}$ is thought to be dependent on the barrier width for electron tunnelling, the position and 
the density of empty electronic states of the adsorbates [48]. It is challenging to determine the contribution of $A_{\text {size }}$ with respect to $A_{\text {adsorbates }}$. Up to now, very little is known about the chemical interface damping and this effect starts to be investigated [48-53]. For the present adsorbates/metal NPs, calculations have shown that $A_{\text {adsorbates }}$ is an important damping channel for surface plasmon [19]. The chemical interface damping results from the strong interaction (chemisorption) between sulfate molecules and gold surface.

\subsection{Electronic structure of metalladsorbates nanoparticles}

The metal mediated-electron delocalization into the adsorbates shell can be seen as a strong mixing of molecular wave functions with electronic bands of the metal substrate. Concerning the geometry of the chemisorption of sulfate molecule onto gold surface, calculations revealed that an oxygen atom of a sulfate molecule is surrounded by three surface gold atoms on face $\left(\begin{array}{lll}1 & 1 & 1\end{array}\right)$ [54]. The LUMOs are mainly formed by sulfur orbitals and the first LUMOs are $\sigma^{*}$ and $\pi^{*}$ molecular orbitals [54]. An electron flow occurs from the oxygen atom of sulfates HOMOs ( $\sigma$ and $\pi$ molecular orbitals) toward empty electronic sp states of the metal. Only the sp bands of the metal is thought to contribute to the interaction since the $d$-bands for gold are fully occupied and lie far below the Fermi level. On the other hand, the response of the metal is a charge transfer toward the sulfate LUMO leading to an increase of charge density around the sulfur atom. The gold-sulfate bonding has both $\sigma^{*}$ and $\pi^{*}$ character [54]. Finally, the decrease of electron density around oxygen atoms and the increase around the sulfur atom can be rationalized by $\sigma$ donation from oxygen atoms toward metal and $\sigma^{*}$ and $\pi^{*}$ back donation from the metal toward the sulfur atom.

\subsection{Femtosecond spectroscopy: optical response of metal: broad-band spectroscopy}

Let us describe the main features of the optical response of metal NPs upon absorption of a laser pulse. The change of absorption measured by ultrafast pumpprobe transient absorption spectroscopy is linked to the transient change of metal optical properties [55]. Indeed, the perturbation in electron distribution affects the dielectric constant of the metal [56,57]. Thus, with this optical technique, it is possible to obtain information in real-time on the thermalization and cooling dynamics of nonequilibrium electrons [58-61]. Fig. 3 shows differential transient absorption spectra recorded with a delay time of $200 \mathrm{fs}$ and $4 \mathrm{ps}$ upon pumping at $480 \mathrm{~nm}$ for $4.2 \mathrm{~nm}$ adsorbates/metal NPs. The dynamics extracted from femtosecond transient absorption spectroscopy may be complicated due to the continuous shape

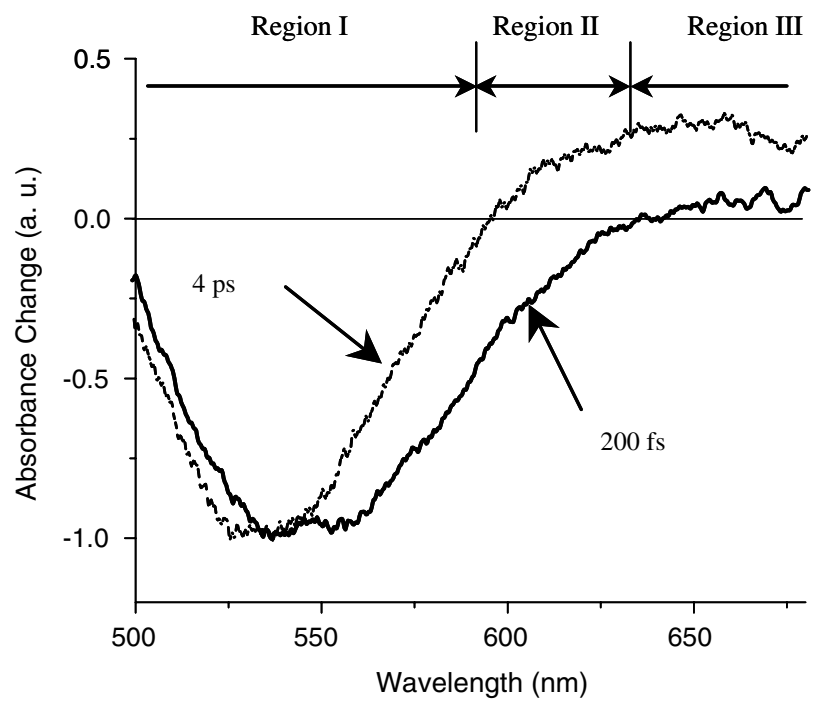

Fig. 3. Differential transient absorption spectra recorded after a delay time of $200 \mathrm{fs}$ and $5 \mathrm{ps}$ after pumping at $480 \mathrm{~nm}$ for metal/adsorbates nanosystems of $4.2 \mathrm{~nm}$ core size.

evolution of the transient absorption spectra [19]. Concerning the distribution of nonequilibrium electron, an important feature is the observation of different spectral signatures for the nonthermal and the thermal distributions, as revealed by broad-band spectroscopy in Fig. 3. Nonthermal distribution is associated to the population of NNEs whereas the internally thermalized is linked to the hot electron gas. The spectral signature of NNEs (nonthermal distribution) consists of a broad featureless bleach (transmission) with a minimum close to surface plasmon resonance in the differential transient absorption spectrum. An important point here is that NNEs do not exhibit any absorption at any wavelength over the whole visible spectrum [62]. Concerning the internally thermalized hot electron gas, the bleach is narrower compared to NNEs and the differential transient spectrum exhibits absorption on both sides of the bleach. In contrast to the spectral signature of NNEs, the shape of hot electron gas has been widely observed for gold NPs [33,34,63-65]. The absorption cross-section of NNEs is much lower compared to the hot electron gas because nonthermal electrons cover a wide energy range with a small electron distribution change whereas hot electron gas covers a narrow energy range with a high electron distribution change. Spectral signatures of NNEs (nonthermal regime) and hot electron gas (thermal regime) obtained here by broad-band spectroscopy are in excellent accordance with calculations based on bulk metal electron kinetics and band-structure modeling in gold NPs [66]. As observed by broad-band spectroscopy, the nonthermal contribution (out of Fermi-Dirac distribution) gives a negative response over the whole probe spectral region while the thermal distribution either exhibits a negative (bleach) or a positive response (wings) responses. It should be noted that the 
shape of the spectral response may change as a function of NPs sizes and that the chemical interface damping can influence the transient spectra.

\subsection{Different spectral regions for the metal response}

The spectral map of nonequilibrium electrons can be divided in three regions as illustrated in Fig. 3. Within region I at the surface plasmon resonance, the overlap of NNEs and hot electron gas responses can make the response complex within this probe region, where a negative signal is observed at any time of process (see Figs. 5 and 6). The absorption cross-section of NNEs is much lower compared to the hot electron gas [55]. These absorption cross-section differences at the early stage can lead to a mutual compensation of the absorbance changes by the simultaneous recovery of the bleach due to the decay of nascent electrons and the increase of the bleach linked to the formation of the hot electron gas. As a result, a fast bleach is formed (not necessary within the instrument response) followed by an exponential recovery until the baseline. Within region II at the red side of surface plasmon resonance, the signal exhibits a change of sign, which is expected in view of the transient absorption spectra. The signal exhibits within this region a bleach (negative signal), which is formed within the instrument response (this bleach reflects the instantaneous creation of NNEs) followed by an exponential recovery to give a positive signal, which further decay to baseline. The change of sign in region II is directly linked to the slow internal thermalization observed with the metal/adsorbates NPs. Within the last region III, only a positive signal is observed with a rise slower than the instrumental response. This transient absorption (wing at the red side of resonance) is linked to the response of hot electron gas. Since the absorption on the red side of the bleach is directly linked to the thermal distribution [55], it is possible to follow the formation of the hot electron gas with the rise of the signal [19].

\subsection{Choice of probe wavelengths to investigate the hot electron cooling}

In previous studies, electron-phonon coupling dynamics were probed at the minimum of the surface plasmon bleach of NPs in region I $[27,28]$ or far off the resonance in region III [35]. The choice of the probe wavelength for the present work to investigate the hot electron cooling process was based on the following observations: From calculations with gold metal, it is known that the response of the thermal distribution dominates in region I at the Fermi energy [55] $\left(E_{\mathrm{F}}=\right.$ $2.4 \mathrm{eV}$ for gold), which is very close to the surface plasmon resonance of these NPs. The response of nonthermal electrons is much weaker in this spectral region

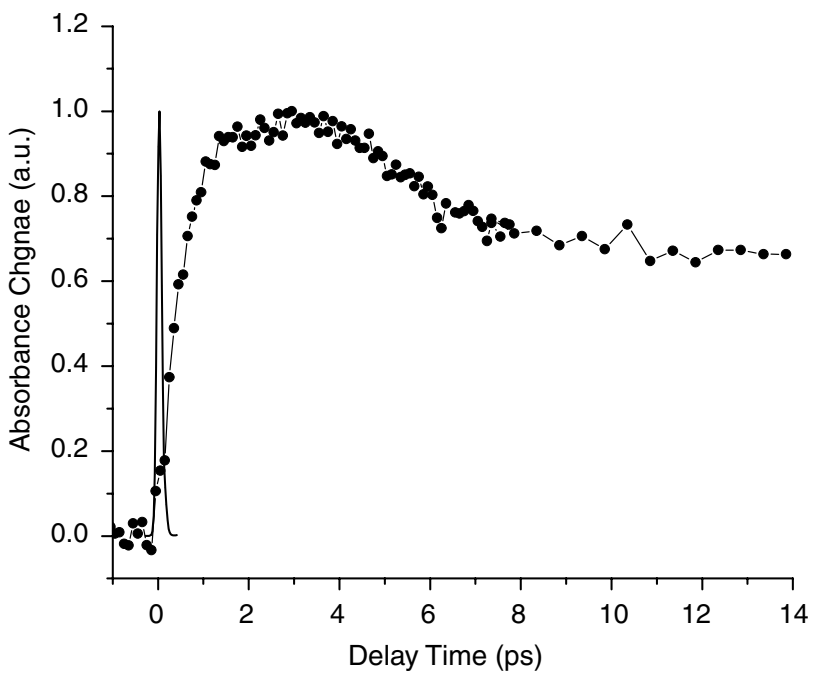

Fig. 4. Transient absorption data after pumping at $535 \mathrm{~nm}$ and probing at $625 \mathrm{~nm}$ (spectral region III, off resonance) for $2.5 \mathrm{~nm}$ adsorbates/metal nanosystems.

[55]. Arbouet et al. have monitor electron-phonon coupling interaction far off the surface plasmon resonance, at the red side of the bleach [35]. Transient thermomodulation transmitivity studies have shown that the response far off resonance (out of region I) directly reflects the nonthermal electron distribution at early stages [55]. This behavior is confirmed in Fig. 4, which shows transient absorption data recorded at $625 \mathrm{~nm}$ (in region III). The signal exhibits a slow rise with a short plateau around $2.5 \mathrm{ps}$ followed by a fast and a slow decay until the baseline. Other NPs sizes exhibit a similar behavior, however with slower rise as the NPs size decrease [40]. Therefore, due to the complex shape of the signal in this region, probe wavelengths out off resonance (out of region I) could not be selected to monitor the hot electron cooling process. In summary, the hot electron energy loss process will be probed in region I, at the plasmon resonance (also Fermi energy) because (i) the response of hot electron gas (thermal distribution) dominates (ii) other regions II and III are highly sensitive to nonthermal distribution (NNEs) (iii) consequence of (ii), the signal exhibits complex behavior (slow rise and plateau) making very difficult the extraction of an accurate value of electron-phonon coupling times in region III off-resonance.

\subsection{Conditions to extract electron-phonon coupling rate}

As the purpose of this work is to compare dynamics of different NPs sizes, it is important to find experimental conditions where the rates are independent on external parameters. Prior embarking on ultrafast spectroscopic measurements on the hot electron cooling process, let us first present the parameters that govern the dynamics e-ph interaction in accordance with the 
widely used TTM. Different methods have been employed to extract the electron-phonon coupling constant. The hot electron cooling process can be divided in two regimes: (i) the so-called high and (ii) the low perturbation regime. The frontier between the two regimes is given by the rise of electronic temperature $\Delta T_{\mathrm{e}}$, i.e., the photonic energy deposited in each nanoparticle. The low-perturbation regime is thought to be reached for $\Delta T_{\mathrm{e}}$ values below $200 \mathrm{~K}$ [68].

(i) A direct consequence of the TTM process is the dependence of the hot electron cooling rates on electronic temperature within the high perturbation regime. This comes from the fact that electronic heat capacity depends on electron temperature with the relation $C_{\mathrm{e}}=\gamma T_{\mathrm{e}}\left(\gamma=66 \mathrm{~J} \mathrm{~m}^{-3} \mathrm{~K}^{-2}\right.$ for gold $)$. As a result, hot electron cooling rates are dependent on electron temperature rise $\Delta T_{\mathrm{e}}$, and therefore on pump fluence. Within the high perturbation regime, the hot electron cooling time is given by $\tau_{0}=\gamma\left(T_{0}+\Delta T_{\mathrm{e}}\right) / g$ with $T_{0}$ the initial temperature. If the cooling lifetimes vary linearly with pump fluences until zero pump fluence, the intercept gives the lifetime for the extrapolation to zero pump fluence. Within this approach, it could be useful to check if lowest measurable value is close to the one obtained with the extrapolation.

(ii) Another approach consists of performing measurements at very low pump fluence in a regime where the hot electron cooling time becomes independent on pump fluence. Within the low perturbation regime $\Delta T_{\mathrm{e}} \ll T_{0}$, the electron-phonon coupling constant can be directly extracted from the measured hot electron lifetimes since then the constant is reduced to $\tau_{0}=\gamma T_{0} / g$. This regime is thought to be reached with an average of one absorbed photon per nanoparticle but the access is made difficult by the low signal-to-noise ratio. Nevertheless, we have used both methods to extract the effective hot electron cooling time constants for the comparison of NPs sizes. However as pointed out above, the approach of the problem could be more complicated compared to "naked NPs" (no influence of the environment) [35] because the assumptions of TTM are transgressed due to the long-lived nonthermal regime observed for the metal/adsorbates nanosystems [19]. The most important point for the present purpose is that the dynamics depend only on the intrinsic properties of metal/adsorbates NPs. In other words, the independence of the dynamics on external parameters allows a direct comparison between the different sizes.

Fig. 5A shows transient absorption data recorded with a probe wavelength at $530 \mathrm{~nm}$ for different pump fluences at $480 \mathrm{~nm}$ and adsorbates/metal nanosystem sizes of $4.2,8$ and $9.2 \mathrm{~nm}$. A pump fluence dependence is observed as expected from the TTM, indicating that the measurements are performed within the high perturbation regime. Fig. 5B shows the hot electron cooling lifetimes as a function of pump fluence for different sizes.
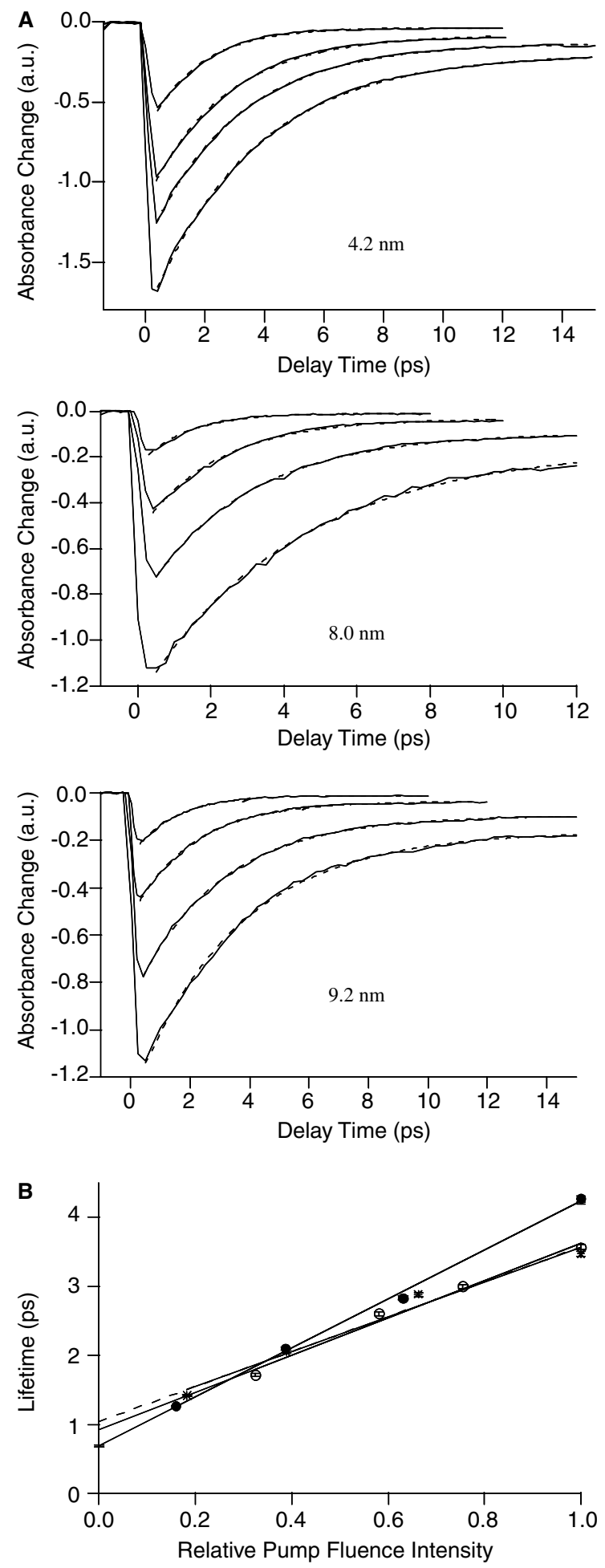

Fig. 5. (A) Transient absorption change for adsorbates/metal nanosystems of $9.2,8.0$ and $4.2 \mathrm{~nm}$ after pumping at $480 \mathrm{~nm}$ and probing at $535 \mathrm{~nm}$ with different pump fluences. (B) Hot electron cooling lifetimes as function of pump fluences for NPs sizes of 9.2, 8 and $4.2 \mathrm{~nm}$ extracted from data obtained after pumping at $480 \mathrm{~nm}$ and probing at $535 \mathrm{~nm}$.

The zero pump fluence extrapolation approach with the intercept of the slope gives values of $(1.05 \pm 0.15)$, $(0.92 \pm 0.15),(0.69 \pm 0.08) \mathrm{ps}$ for NPs sizes of $4.2,8$ 
and $9.2 \mathrm{~nm}$, respectively. The extrapolation indicates a lower hot electron cooling rate for the smallest nanoparticles. The hot electron cooling time obtained for the $9.2 \mathrm{~nm}$ NPs is very close to those for gold NPs in solution $[32,67]$. However, due to the uncertainties for the value of electron-phonon coupling constant, the dependence on pump fluence and the narrow range of NPs sizes studied, it could be useful to perform measurements in the weak perturbation regime in order to carefully compare the dynamics with different sizes. To check if the zero pump fluence corresponds to the lowest measurable lifetimes, we performed additional experiments by pumping and probing at $535 \mathrm{~nm}$ with a larger size range from 1.7 to $9.2 \mathrm{~nm}$.

One-colour pump-probe experiments allowed improving the signal-to-noise ratio of our set-up to explore the weak perturbation regime. Fig. 6 shows femtosecond data for 1.7, 2.5 and $4.2 \mathrm{~nm}$ NPs sizes with different pump fluences. The superposition of the normalized recovery curves below a pump fluence threshold value indicates that rates of hot electron cooling are independent on pump fluence. Fig. 7 shows that the hot electron cooling lifetimes for NPs of $4.2 \mathrm{~nm}$ also become pump fluence independent below an intensity of $100 \mathrm{~nJ}$ per pulse, indicating that the measurements are performed within the very low perturbation regime (less than one absorbed photon per nanoparticles in average). The same phenomenon is observed for $2.5 \mathrm{~nm} \mathrm{NPs}$, the weak perturbation regime is reached with a higher pump fluence due to the fact that the number of $2.5 \mathrm{~nm}$ NPs in the matrix is about five times higher compared to $4.2 \mathrm{~nm}$ NPs. For the $1.7 \mathrm{~nm} \mathrm{NPs}$, the weak perturbation regime is easily attained but the signal to noise ratio is low due to the weak response (surface plasmon is highly damped). The minimum electronic temperature rise per nanoparticule is fixed by the absorption of a photon. For the smallest NPs $(1.7 \mathrm{~nm})$, even with an average of one absorbed photon per nanoparticle, $\Delta T_{\mathrm{e}}$ may be high due to the confinement of the photonic energy [29]. However, this effect does not affect the comparison between NPs sizes, as checked by Arbouet et al. [35] with the dependence on pump photon energy. Finally, energy-loss dynamics for different NPs sizes can be obtained within the same conditions. For the fitting procedure, all curves are fitted within the same manner with an exponential decay plus a baseline. Time constants in the low perturbation regime depend on intrinsic properties of the adsorbates/metal nanoparticles and we can now dress the size behavior for the critical size range. Fig. 8 showing hot electron cooling lifetimes as a function metal/adsorbates NPs sizes reveals a decrease of energy loss rate as the NPs sizes decrease. The values $\tau_{\mathrm{e}-\mathrm{ph}}^{0}$ obtained for the largest gold NP is very close to the lifetime reported for similar gold NPs sizes [35] or bulk materials $[68,69]$. To summarize the experimental results, measurements performed in the weak perturba-
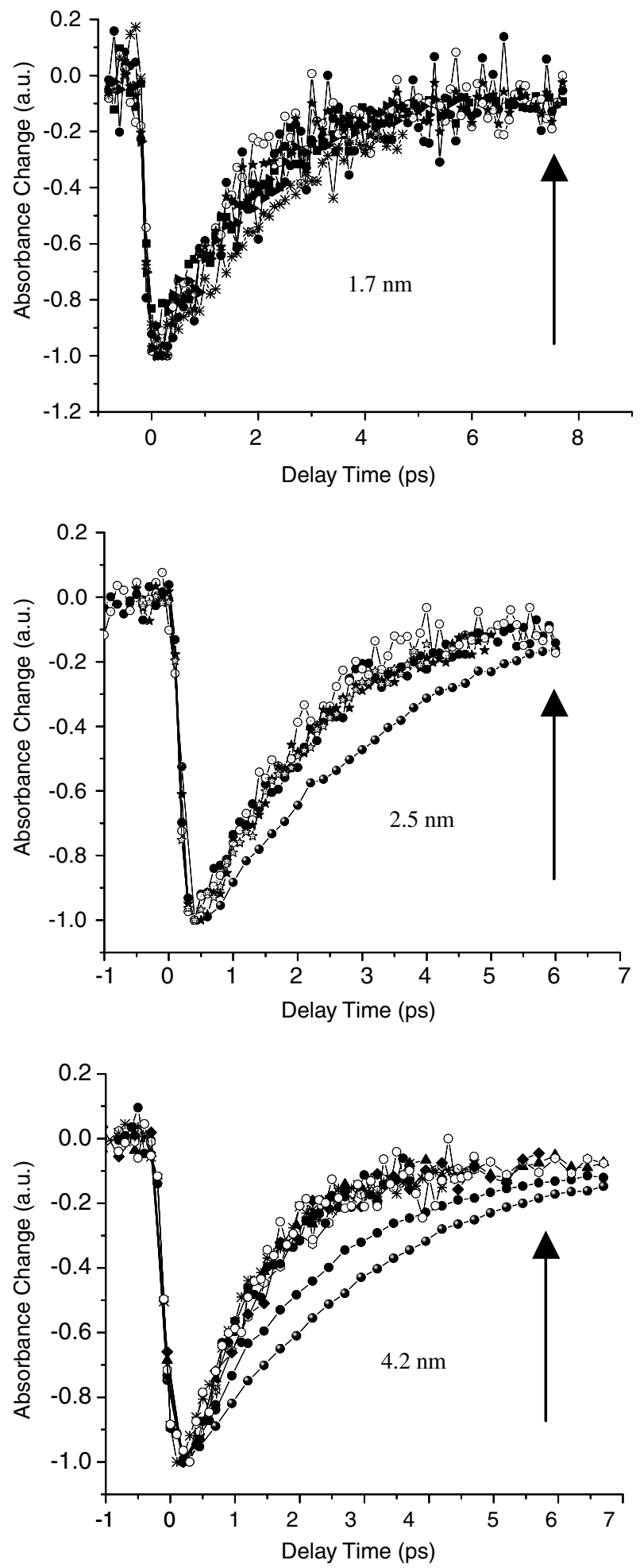

Fig. 6. Transient absorption change for adsorbates/metal nanosystems sizes of 4.2 and $2.5 \mathrm{~nm}$ after pumping and probing at $535 \mathrm{~nm}$. Pump fluence of $340,144,50,36,25,20,15 \mathrm{~nJ} /$ pulse for $4.2 \mathrm{~nm}, 300,170,143$, $130,100,85 \mathrm{~nJ} /$ pulse for $2.5 \mathrm{~nm}$ and $210,180,150,100,80$ and 50 $\mathrm{nJ} /$ pulse for $1.7 \mathrm{~nm}$. The arrows indicate the decrease of pump fluence.

tion regime have shown that hot-electron cooling rates decrease with metal/adsorbates nanosystem sizes. When the surface to volume ratio exceeds $30 \%$, surface effects 

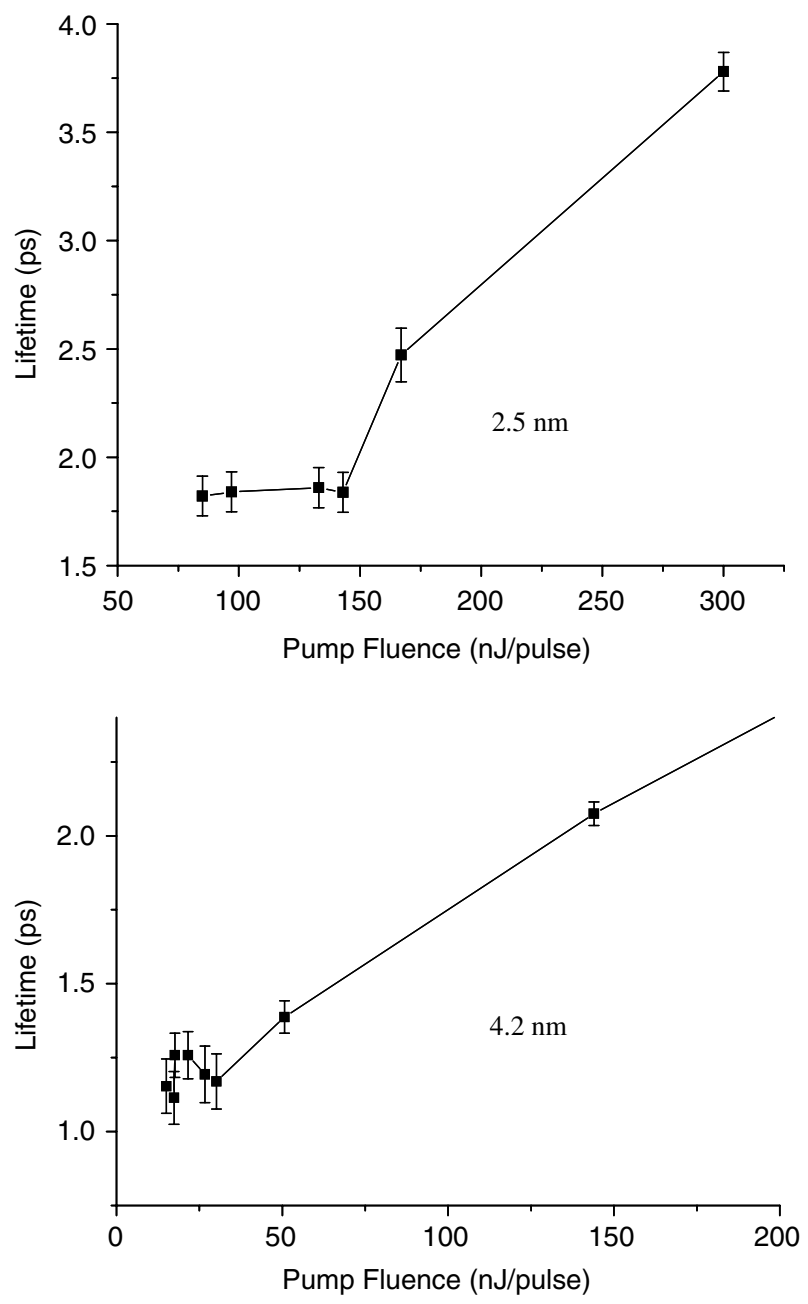

Fig. 7. Examples of hot electron cooling lifetimes as a function of pump fluences for NPs of 2.5 and $4.2 \mathrm{~nm}$.

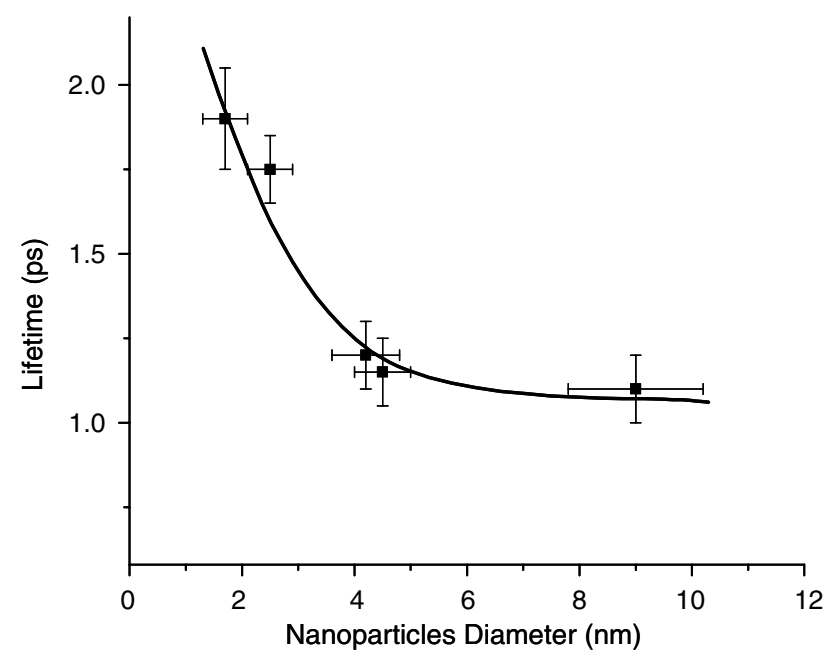

Fig. 8. Hot electron cooling lifetimes as a function of adsorbates/metal NPs sizes. The lifetimes were extracted from data obtained within the weak-perturbation regime under the same conditions. The solid line is a guide for the eyes. become more important and electron-phonon coupling time as long as $1.9 \mathrm{ps}$ is observed for the smallest metal/adsorbates nanosystems.

\subsection{Effect of incomplete internal thermalization on hot electron cooling dynamics}

In the following section, an explanation for the delayed hot electron cooling process is proposed based on electron distribution changes in metal and the presence of a long-lived nonthermal regime. Experimental evidence for an incomplete internal thermalization (nonthermal regime) comes from the data obtained by probing in region III where a slow rise due to the finite time for the formation of the hot electron gas is clearly observed [19]. An example of this behavior is shown in Fig. 4 for the $2.5 \mathrm{~nm}$ NPs where the rise of the signal probed at $625 \mathrm{~nm}$ directly reflects the temporal evolution from a nonthermal to a thermal distribution $[19,55]$. The size dependence behavior of internal thermalization is beyond the scope of this paper, however, it is worthwhile noting that this process becomes longer as the NPs size decrease [40]. In these metal/adsorbates NPs, the internal thermalization process to form the hot electron gas consists of e-e scattering and e-MV interactions (electron-molecular vibration coupling). Nonthermal electrons spread the excess energy by collision with "cold" electrons and by excitation of adsorbates vibrational modes. Early works have already shown that a nonthermal distribution can considerably slow down the hot electron energy-loss dynamics in bulk gold $[16,70]$. The electron-phonon coupling rate can be defined as the average rate of phonon emission [70]. During the establishment of a Fermi-Dirac distribution, the average rate of electron-phonon coupling is expected to increase exponentially with time. Indeed, during the internal thermalization process, there is a continuous increase of electron distribution changes during the evolution from a nonthermal to a thermal regime. Fig. 9 illustrates the electron distribution changes as a function of electron energy for a nonthermal and a thermal distribution. The main information from Fig. 9 is the increase of the number of electrons out of equilibrium during the establishment of a Fermi-Dirac distribution. Since the internal thermalization does not occur within the laser pulse duration in metal/ adsorbates nanosystems, only a fraction of electrons (population of NNES) is out of equilibrium. And, as the population size of NNEs is much lower compared to the hot electron gas, the probability of electron-phonon interaction is significantly decreased within the nonthermal regime compared to the thermal regime. As a result, time rates of hot electron cooling are reduced for the present systems.

It is worthwhile noticing that the TTM does not account for the existence of a possible nonthermal 


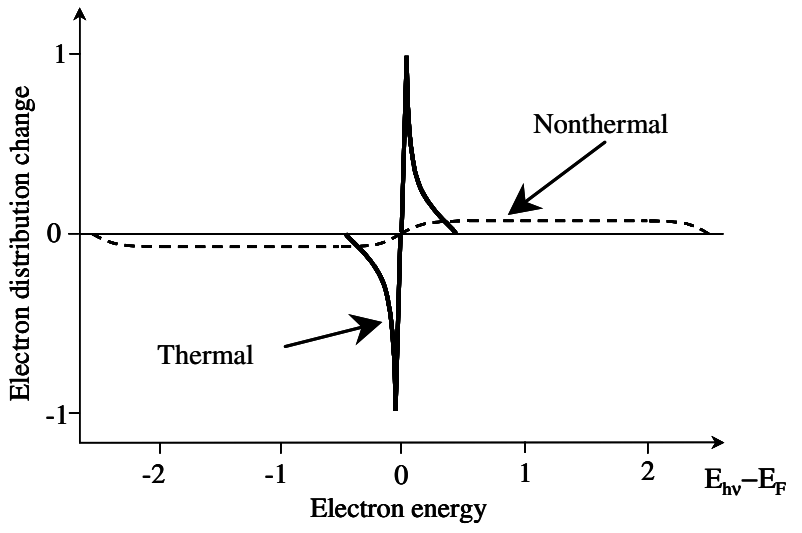

Fig. 9. Qualitative picture illustrating the change of electron distribution as a function of electron kinetic energy in a metal for NNEs size population (nonthermal regime) and for hot electron gas (thermal regime) assuming intraband electronic transition with equal probability and a pump wavelength at $535 \mathrm{~nm}$. The change of electron distribution reflects the population of electrons out of equilibrium, which is very low at time zero (NNEs) and increase as NNEs spread the kinetic energy by e-e collisions with equilibrium (cold) electrons making kinetic energy range much more narrow for hot electron gas at the end of internal thermalization (after [55]).

regime for times longer than the laser pulse duration. Indeed, when the electronic temperature is established, all nonthermal electrons are supposed to be thermalized within the hot electron gas. This situation corresponds to the thermal distribution with a high electron distribution change over a narrow energy range as shown in Fig. 9. Therefore, at the end of the internal thermalization, the number of electrons out of equilibrium able to emit a phonon is constant and the electron-phonon coupling constant can be defined since the coupling strength does not evolve with time [16,70]. In summary, the hot electron cooling process is delayed because the average electron-phonon coupling strength increases as long as the internal thermalization is in progress. For the present systems, the internal thermalization can be as long as $1 \mathrm{ps}$ for the smallest sizes [40].

\subsection{Comparison with other size behavior investigations with metal NPs}

It is interesting to notice that for gold and silver NPs not influenced by the environment, the size dependence on electron-electron scattering rate [66] follows the same trend as the electron-phonon coupling rate [35]. Both e-e $[66,71]$ and e-ph interaction rates in $\mathrm{Ag}$ and $\mathrm{Au}$ NPs increase with the decrease of naked NPs sizes [35]. The information resulting from the present work concerning the link between e-e and e-ph scattering processes is comparable with the observation of the size behavior of NPs reported for Ag and Au NPs [35]. The main difference is that both internal thermalization and electron-phonon coupling rates for the present adsorbates/metal nanosystems exhibit the opposite trend with respect to $\mathrm{Ag}$ and $\mathrm{Au}$

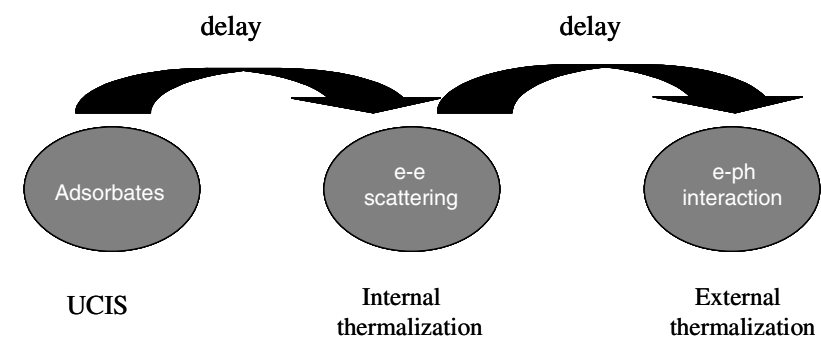

Fig. 10. Simplified schematic picture illustrating the dynamical interplay between the adsorbates, phonons and electrons in adsorbates/ metal nanosystems. Reduction of internal thermalization by the adsorbates due to ultrafast chemical interface scattering (UCIS). The long resulting nonthermal regime affects the electron-phonon coupling strength. As an effect of the increase of S/V ratio, the role of adsorbates becomes more important as the NPs sizes decrease.

NPs [40]. Let us emphasize the difference between size and surface effects. Size effects in NPs arise from the confinement whereas surface effects are related to the adsorbed chemical species interacting with the metal surface. As a result, for naked NPs only size effects (which are given by bulk properties) may arise in this case. For adsorbates/metal nanosystems bulk properties are not sufficient to understand the results since surface effects emerge from the chemical surrounding medium around the nanoparticle. Therefore, the surface effects governed by the properties of adsorbates may play a crucial role on the dynamics of nonequilibrium electrons. The main difference for sulfate/gold NPs with respect to "naked" gold NPs is the presence of additional channel for nonthermal electron scattering, the bidirectional electron tunneling process, i.e., chemical interface damping [19]. Our results confirm that the internal thermalization process governs the subsequent dynamical events of nonequilibrium electron dynamics. According to our findings for adsorbates/metal NPs, it is possible to drawn the simplified schematic picture illustrating the interconnection between the different processes of electron redistribution and energy dissipation involving the adsorbates, nonequilibrium electrons and phonons. The proposed scheme shown in Fig. 10 is the following: Adsorbates strongly affect the dynamics of internal thermalization and give rise to a long-lived nonthermal regime. Because of the crucial role played by the adsorbates, the effect of the nonthermal regime becomes more important as the surface to volume ratio of NPs increases. Due to the reduction of the electron-phonon coupling strength within the nonthermal regime, the hot electron cooling process is slowed down compared to the naked gold NPs or bulk system as the size decreases.

\section{Conclusion}

The size dependence of hot electron cooling rate in gold NPs wrapped in a shell of sulfate has been 
investigated by ultrafast transient absorption spectroscopy. Comparison of hot electron cooling times under the same conditions for adsorbates/metal NPs in size range of 1.7-9.2 $\mathrm{nm}$ reveals a decrease of energy loss rate with NPs sizes. This behavior is attributed to the incomplete internal thermalization observed in these systems, which slows down the hot electron cooling process. A chain of complex dynamical events takes place in the adsorbates/metal nanosystem where adsorbates first slow down the internal thermalization (e-e scattering) during the early stages of photonic energy dissipation. Therefore, the rate of phonons emission is initially very low during the nonthermal regime due to the small population size of electrons out of equilibrium (NNEs) able to interact with phonons compared to the thermal regime (hot electron gas). These observations suggest that the retardation of internal thermalization leads to a decrease of the strength of electron-phonon coupling and that the dynamics can hardly be described within the frame of the two-temperature model. Furthermore, our results show that surface effects (adsorbates) dominate size effects for the control of dynamical behavior of nonequilibrium electrons in metal/asorbates nanosystems. The energy flow between metal and adsorbates is mediated by the charge resonance process (chemical interface damping). Finally, the delayed hot electron cooling induced by the nonthermal distribution highlights the strong effect of adsorbates on nonequilibrium electrons dynamics in metal NPs.

\section{Acknowledgments}

C.B. gratefully acknowledges Pascal Comte and Raphael Charvet for the preparation of the titanium dioxide matrix, Valérie Devaud for the technical assistance and J.-E. Moser and B. Wenger for the help with the femtosecond laser set-up. The authors acknowledge the Centre Interdépartemental de Microscopie (EPFL) for the TEM pictures. The present work is financed by the "Fonds National Suisse de la Recherche Scientifique" and the "Office Fédérale de l'Education et de la Science (project C02.0010)". The Laboratoire d'Electrochimie Physique et Analytique is part of the TMR network SUSANA (Supramolecular Self-Assembly of Interfacial Nanostructures).

\section{References}

[1] A.M. Kuznetsov, Charge Transfer in Physics, Chemistry and Biology, Gordon and Breach, New-York, 1995.

[2] H.H. Girault, Analytical and Physical Electrochemistry, Marcel Dekker, 2004.

[3] R.J.D. Miller, G.L. McLendon, A.J. Nozik, W. Schmickler, F. Willig, Surface Electron-Transfer Processes, VCH, New-York, 1995.
[4] X.-Y. Zhu, J. Phys. Chem. B 108 (2004) 8778.

[5] H. Petek, H. Nagano, M.J. Weida, S. Ogawa, J. Phys. Chem. B 105 (2001) 6767.

[6] M. Bonn, S. Funk, C. Hess, D.N. Denzler, C. Stampf, M. Scheffler, M. Wolf, G. Ertl, Science 285 (1999) 1042.

[7] W. Ho, J. Chem. Phys. 117 (2002) 11033.

[8] H. Ishii, K. Sugiyama, E. Ito, K. Seki, Adv. Mater. 11 (1999) 605.

[9] D. Cahen, A. Kahn, Adv. Mater. 15 (2003) 271.

[10] J.P. Jiang, K. Bosnick, M. Maillard, L. Brus, J. Phys. Chem. B 107 (2003) 9964.

[11] C. Joachim, J.K. Gimzwski, A. Aviram, Nature 408 (2000) 541.

[12] A. Nitzan, M.A. Ratner, Science 300 (2003) 1384.

[13] It could be useful to point out that all the following processes are equivalent and describe the same phenomenon: internal thermalisation, electron-electron scattering, build-up of Fermi-Dirac electron distribution, hot electron thermalization, formation of a hot electron gas, establishment of an electronic temperature. The hot electron cooling is also called the external thermalization, nonequilibrium energy-loss process or electron-phonon interaction process.

[14] M.I. Kaganov, I.M. Lifshitz, L.V. Tanarov, Sov. Phys. JETP 4 (1957) 173.

[15] S.I. Animisov, B.L. Kapeliovich, T.L. Perel'man, Sov. Phys. JETP 39 (1974) 375.

[16] R.H.M. Groeneveld, R. Sprik, A. Lagendijk, Phys. Rev. B 51 (1995) 11433.

[17] M. Lisowski, P. Loukakos, U. Bovensiepen, J. Stahler, C. Gahl, M. Wolf, Appl. Phys. A 78 (2004) 165.

[18] B. Rethfeld, A. Kaiser, M. Vicanek, G. Simon, Phys. Rev. B 65 (2002).

[19] C. Bauer, J.-P. Abid, D. Fermin, H.H. Girault, J. Chem. Phys. 120 (2004) 9302.

[20] R.R. Cavanagh, D.S. King, J.C. Stephenson, T.F. Heinz, J. Phys. Chem. 97 (1993) 786.

[21] J.W. Gadzuk, Chem. Phys. 251 (2000) 87.

[22] D. Menzel, R. Gomer, J. Chem. Phys. 41 (1964) 3311.

[23] P.A. Redhead, Can. J. Phys. 42 (1964) 886.

[24] P.R. Antoniewicz, Phys. Rev. B 21 (1980) 3811.

[25] R.J. Deliwala, R.J. Finlay, T.H. Goldmann, T.H. Her, W.D. Mieher, E. Mazur, Chem. Phys. Lett. 242 (1995) 617.

[26] T.-H. Her, R.J. Finlay, C. Wu, E. Mazur, J. Chem. Phys. 108 (1998) 8595.

[27] S. Link, M.A. El-Sayed, J. Phys. Chem. B 103 (1999) 8410.

[28] J.H. Hodak, A. Henglein, G.V. Hartland, J. Phys. Chem. B 104 (2000) 9954.

[29] C. Voisin, N. Del Fatti, D. Christofilos, F. Vallée, J. Phys. Chem. B 105 (2001) 2264.

[30] N. Del Fatti, F. Vallee, C. R. Phys. 3 (2002) 365.

[31] G.V. Hartland, Int. J. Nanotechnol. 1 (2004) 307.

[32] J.H. Hodak, A. Henglein, G.V. Hartland, J. Chem. Phys. 112 (2000) 5942.

[33] T.S. Ahmadi, S.L. Logunov, M.A. El-Sayed, J.T. Khouri, R.L. Whetten, J. Phys. Chem. B 101 (1997) 3713.

[34] S. Link, C. Burda, Z.L. Wang, M.A. El-Sayed, J. Chem. Phys. 111 (1999) 1255.

[35] A. Arbouet, C. Voisin, D. Christofilos, P. Langot, N. Del Fatti, F. Vallée, J. Lerme, G. Celep, E. Cottancin, M. Gaudry, M. Pellarin, M. Broyer, M. Maillard, I.M. Pilen, M. Treguer, Phys. Rev. Lett. 90 (2003) 177401.

[36] S. Link, A. Furube, M.B. Mohamed, T. Asahi, H. Masuhara, M.A. El-Sayed, J. Phys. Chem. B 106 (2002) 945.

[37] M.B. Mohamed, T.S. Ahmadi, S. Link, M. Braun, M.A. ElSayed, Chem. Phys. Lett. 343 (2001) 55.

[38] S. Link, D.J. Hathcock, B. Nikoobakht, M.A. El-Sayed, Adv. Mater. 15 (2003) 393.

[39] S.L. Westcott, R.D. Averitt, J.A. Wolfgang, P. Nordlander, N.J. Halas, J. Phys. Chem. B 105 (2001) 9913. 
[40] C. Bauer, J.-P. Abid, Hubert. H. Girault, submitted.

[41] S. Pelet, M. Gratzel, J.E. Moser, J. Phys. Chem. B 107 (2003) 3215.

[42] U. Kreibig, M. Vollmer, Optical Properties of Metal Clusters, Springer, Berlin, 1995.

[43] K.L. Kelly, E. Coronado, L.L. Zhao, G.C. Schatz, J. Phys. Chem. B 107 (2003) 668.

[44] U. Kreibig, Z. Phys. 234 (1970) 307.

[45] B.N.J. Persson, Surf. Sci. 283 (1993) 153.

[46] H. Hövel, S. Fritz, A. Hilger, U. Kreibig, Phys. Rev. B 48 (1993) 18178.

[47] T. Linnert, P. Mulvaney, A. Heinglein, J. Phys. Chem. 97 (1993) 679.

[48] U. Kreibig, M. Gartz, A. Hilger, Ber. Bunsenges. Phys. Chem. 101 (1997) 1593.

[49] A. Hilger, M. Tenfelde, U. Kreibig, Appl. Phys. B 73 (2001) 361.

[50] J. Bosbach, C. Hendrich, F. Stietz, T. Vartanyan, F. Trager, Phys. Rev. Lett. 89 (2002).

[51] C. Heindrich, J. Bosbach, F. Stietz, F. Hubenthal, T. Vartanyan, F. Träger, Appl. Phys. B 76 (2003) 869.

[52] A. Pinchuk, U. Kreibig, New J. Phys. 5 (2003) 151.

[53] A. Pinchuk, U. Kreibig, A. Hilger, Surf. Sci. 557 (2004) 269.

[54] E.M. Patrito, P. Paredes Olivera, H. Sellers, Surf. Sci. 380 (1997) 264.

[55] C.K. Sun, F. Vallée, L.H. Acioli, E.P. Ippen, J.G. Fujimoto, Phys. Rev. B 50 (1994) 15337.

[56] R. Rosei, Phys. Rev. B 10 (1974) 474.

[57] R. Rosei, F. Antonangeli, U.M. Grassano, Surf. Sci. 37 (1973) 689.

[58] H.E. Elsayed-Ali, T.B. Norris, M.A. Pessot, G.A. Mourou, Phys. Rev. Lett. 58 (1987) 1212.

[59] S.D. Brorson, J.G. Fujimoto, E.P. Ippen, Phys. Rev. Lett. 59 (1987) 1962.
[60] R.H.M. Groeneveld, R. Sprik, A. Lagendijk, Phys. Rev. Lett. 64 (1990) 784.

[61] T. Juhasz, H.E. Elsayed-Ali, G.O. Smith, C. Suarez, W.E. Bron, Phys. Rev. B 48 (1993) 15488.

[62] The transient absorption observed for wavelengths longer than $630-640 \mathrm{~nm}$ is due to the fact that the transient spectrum was not recorded immediately after the time zero, therefore the internal thermalization has already started. The crossing point of the transient spectrum with the baseline shifts toward the blue with increasing delay time.

[63] T.S. Ahmadi, S.L. Logunov, M.A. El-Sayed, J. Phys. Chem. B 100 (1996) 8053.

[64] M. Perner, P. Bost, G. Lemmer, G. von Plessen, J. Feldmann, U. Becker, M. Mennig, M. Schmitt, H. Schmitt, Phys. Rev. Lett. 78 (1997) 2192.

[65] H. Inoue, K. Tanaka, I. Tanahashi, K. Hirao, Phys. Rev. B 57 (1998) 11334

[66] C. Voisin, D. Christofilos, P.A. Loukakos, N.D. Del Fatti, F. Vallée, J. Lermé, M. Gaudry, E. Cottancin, M. Pellarin, M. Broyer, Phys. Rev. B 69 (2004) 195416.

[67] J.H. Hodak, I. Martini, G.V. Hartland, Chem. Phys. Lett. 284 (1998) 135.

[68] N. Del Fatti, C. Voisin, M. Achermann, S. Tzortakis, D. Christofilos, F. Vallée, Phys. Rev. B 61 (2000) 16956.

[69] J. Hohlfeld, S.S. Wellershoff, J. Gudde, U. Conrad, V. Jahnke, E. Matthias, Chem. Phys. 251 (2000) 237.

[70] R.H.M. Groeneveld, R. Sprik, A. Lagendijk, Phys. Rev. B 45 (1992) 5079.

[71] C. Voisin, D. Christofilos, N.D. Fatti, F. Vallee, B. Prevel, E. Cottancin, J. Lerme, M. Pellarin, M. Broyer, Phys. Rev. Lett. 85 (2000) 2200. 